\title{
Challenging paradigms in tumour drug delivery
}

\author{
A collective effort from materials scientists, life scientists and clinicians is required to systematically address \\ fundamental questions in the mechanisms of nanoparticle delivery in order to overcome the hurdles in translating \\ nanomedicines for tumour therapy.
}

O ver the past three decades, there has been no shortage of nanomedicines developed for cancer therapy, with increasingly complex formulations being produced at an incredible rate. However, only ten nanoparticle-based nanomedicines have been approved for clinical use in cancer therapy by the Food and Drug Administration and European Medicines Agency ${ }^{1}$. Of the nanomedicines that reach phase III clinical trials, as little as $14 \%$ have been successful at demonstrating efficacy ${ }^{1}$. Doxil/Caelyx was the first approved nanomedicine for breast cancer in 1995 and since then, other nanomedicines such as Abraxane, and Vyxeos have been developed for cancers including metastatic breast cancer, pancreatic adenocarcinoma and leukaemia. There is certainly no doubt that nanoparticles have been beneficial in the delivery of cancer therapeutics. It is now known that the use of nanoparticles as carriers for chemotherapeutics has enormously enhanced patient tolerance by limiting systemic toxicity ${ }^{2}$. Regrettably, however, the high attrition rate has led to some scepticism about the potency of nanomedicines.

The poor efficacy of nanomedicines has been attributed to factors such as poor accumulation of nanoparticles in tumours as well as poor pharmacokinetics ${ }^{1}$. After three decades, and with the enormous quantity of data published, it is perhaps time to appraise the field and attempt to overcome the hurdles in clinical translation. Warren Chan and colleagues recently carried out an analysis of the published nanomedicine literature over a ten-year period (2005-2015) and found that an average of $0.7 \%$ of nanoparticles that were administered systemically reach solid tumours ${ }^{3}$. One critique of this analysis is that the $0.7 \%$ noted is a median and undoubtedly masks the greater success rate of some nanoparticles. However, it is still clear that overall, a large number of nanoparticle formulations have had poor delivery efficiency.

One of the mechanisms for the entry of nanoparticles into tumours is the principle of enhanced permeability and

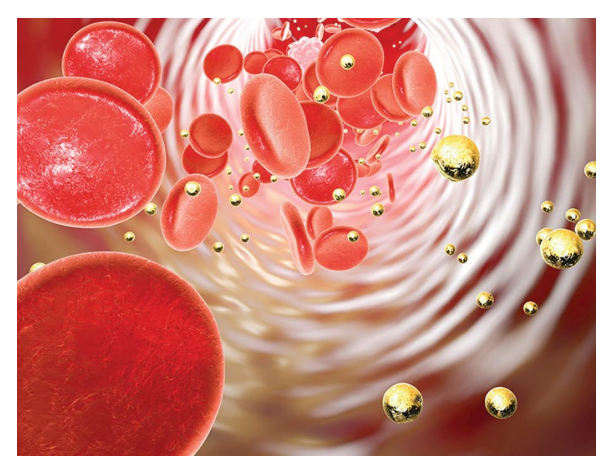

Illustration of nanoparticles administered into the blood stream to target tumours. Credit: Science Photo Library/Alamy Stock Photo

retention (EPR). This principle was first observed by Maeda and colleagues in $1986^{4}$ and it involves the accumulation of macromolecules and particles into tumours, driven by the hyper-permeable vasculature and the lack of a lymphatic drainage system within tumours. The EPR effect has almost been accepted unequivocally by the community and it is only recently that it has been mired in controversy due to claims that it may vary between tumour types. Indeed, recent work by Andresen and colleagues attempted to elucidate liposome retention in a number of tumour types and demonstrated that the EPR effect cannot be considered a general principle due to the heterogeneity in nanoparticle accumulation in the different tumours investigated $^{5}$. A better understanding of nanoparticle extravasation from blood vessels is required (pictured).

In their latest research, an Article in this issue, Warren Chan and colleagues delved deeper into the mechanisms mediating nanoparticle entry into solid tumours. They demonstrated that as much as $97 \%$ of nanoparticles are transported into solid tumours by endothelial cells through an active process of transcytosis. They also demonstrate that, contrary to popular belief in the EPR effect, the frequency of gaps on the endothelial lining is too low to account for the accumulation of nanoparticles into tumours. Chan and colleagues also developed a 'zombie' mouse model, whereby tumour-bearing mice were perfused with a tissue fixative to deactivate any cellular activity while preserving vessel architecture. With this model, they could definitively account for the contribution of passive extravasation through the inter-endothelial gaps since the mice did not have a functional nanoparticle clearance mechanism.

This study has been summarized by David Mooney and Irene de Lázaro in a News \& Views, highlighting that the findings are disruptive and constitute a wake-up call to the community to revisit the long-established paradigms in nanoparticle delivery mechanisms. In a Comment, Shuming Nie and colleagues also discuss that these findings should galvanize researchers to develop techniques that harness the active transcytosis process to enhance delivery efficiency. This could be achieved by employing approaches that utilize tumour-penetrating peptides ${ }^{6}$. Other related approaches have focused on designing polymer conjugates that employ a cationization process that permits adsorption-mediated active transcytosis ${ }^{7}$.

It is important for the community to shift more focus towards fundamental research in order to understand the basic principles of the interaction of nanoparticles with the body. The field should also work more closely with life scientists and oncologists who have a better appreciation of tumour biology and patient specificity. Ultimately, it will take many more bold studies that truly challenge existing paradigms in order to make headway in cancer nanomedicine.

Published online: 24 April 2020 https://doi.org/10.1038/s41563-020-0676-X

\footnotetext{
References

1. He, H., Liu, L., Morin, E. E., Liu, M. \& Schwendeman, A. Acc. Chem. Res. 52, 2445-2461 (2019).

2. Salvioni, L. et al. Cancers 11, 1855 (2019).

3. Wilhelm, S. et al. Nat. Rev. Mater. 1, 16014 (2016).

4. Matsumura, Y. \& Maeda, H. Cancer Res. 46, 6387-6392 (1986)

5. Hansen, A. E. et al. ACS Nano 9, 6985-6995 (2015).

6. Liu, X. et al. J. Clin. Invest. 127, 2007-2018 (2017).

7. Zhou, Q. et al. Nat. Nanotechnol. 14, 799-809 (2019).
} 